\title{
Conceptual Design of an Unmanned Aerial Vehicle for Mars Exploration
}

\author{
Ishan Mishra, Aayush Kumar, and Vanshaj Malhotra
}

\begin{abstract}
Significant technology advances have enabled planetary exploration aircraft to be considered as a viable science platform. These systems fill in a unique planetary science measurement gap, that of the regional scale, near-surface observation while providing a new perspective for planetary discovery. Exploration of Mars using UAV (Unmanned Aerial Vehicle) has been planned for over 25 years by leading space organizations such as NASA. Recent efforts have been able to produce some mature mission and flight system concepts, ready for flight project implementation. There are however siognificant numbers of challenges associated with getting an airplane to fly through the thin, carbon dioxide-rich Martian atmosphere. Traditional aircraft design expertise does not always apply to this sort of vehicle, and geometric, aerodynamic, and mission restrictions result in a restricted viable design space. This paper presents the conceptual approach that was taken to design a UAV capable of performing a VTOL (Vertical Take-Off and Landing) in the atmosphere of Mars. The UAV was designed to participate in the International Planetary Aerial Systems Challenge 2021. The UAV could carry a science payload of up to $5 \mathrm{~kg}$ (weight on mars).
\end{abstract}

Key words — Mars Airplane; Science Payload; UAV; VTOL.

\author{
Abbreviations Used: \\ UAV - Unmanned Aerial Vehicle \\ VTOL - Vertical Take-Off and Landing \\ EP - Extreme Pressure \\ PFPE - Perfluoropolyether \\ PTFE - Polytetrafluoroethylene \\ FEA - Finite Element Analysis \\ NACA - National Advisory Committee for Aeronautics \\ TCS - Temperature Control System \\ RHU - Radioisotope Heating Units \\ CCD - Charge Coupled Device
}

\section{INTRODUCTION}

For at least 50 years, people have imagined winged spacecraft flying through the Martian atmosphere. The Mars Project, written by Wernher von Braun and originally published in the early 1950s. For almost 25 years, considerable attention has been given to the use of an unmanned aerial vehicle (UAV) as a research platform for exploring Mars. From its unique vantage point, a few kilometres above the Martian surface, an autonomous airplane could return unique science measurements over regional-scale distances. By traversing regional-scale distances at near-surface altitude, planetary airplane observations complement and extend orbital and landed measurements while providing a fresh perspective for scientific discovery.

This paper presents the design of an Unmanned Aerial Vehicle capable of VTOL working under the Martian atmosphere. The UAV is a solar-powered wing body design having the main wingspan of $3.6 \mathrm{~m}$ and a root chord of $0.5 \mathrm{~m}$. Based on the DOS SAMARA [1] concept the main wing is divided into 2 sections i.e., the inner and outer sections. The outer section is $1 \mathrm{~m}$ long and rotates to generate sufficient thrust for VTOL operations. With a full payload of about 12 $\mathrm{Kgs}$, the UAV is capable of traveling $10 \mathrm{Kms}$ having a velocity of $16.66 \mathrm{~m} / \mathrm{s}$ during the cruise while flying at a max altitude of $7 \mathrm{~km}$ from the ground. A propeller located at the back of the plane provides sufficient thrust to counteract the drag forces, putting the plane in steady and levelled flight conditions.

\section{REQUIREMENTS AND CONSIDERATIONS}

The first and foremost consideration is the nature of the Martian atmosphere. Atmospheric density near the surface of Mars is roughly equivalent to an altitude of 100,000 feet on Earth. It is difficult to create enough lift to maintain the airplane's weight in such a thin atmosphere, thus wing loading must be kept to a minimum.

Owing to the reduced gravity on Mars the weight of the airplane is roughly $38 \%$ of its weight on earth. Nonetheless, to attain a reduced wing loading, a considerable focus must be made on reducing airframe and system weight.

Because of the low air density, the cruise velocity is increased, but the flight Reynolds number is reduced. Because of the lower speed of sound on Mars than on Earth, transonic aerodynamic effects are encountered at lower flight speeds. To reduce significant lift losses and/or drag increases associated with low Reynolds number, high Mach number flying circumstances, special consideration was necessary for the aerodynamic design.

Thrust generation is further complicated by low atmospheric density. The lower the density, the less mass available for momentum transfer and the less thrust generated for a given size propulsion system.

\section{MISSION LAYOUT}

Extensive trade studies and design cycles were performed to develop a low-risk mission architecture. The resultant 
architecture enabled the satisfaction of the mission requirements with the use of a basic, low-risk flying system design.

The UAV will fly a pre-planned $5 \mathrm{~km}$ route from a vantage point 7-8 $\mathrm{km}$ above the ground and then return to the base. The UAV could carry a science payload weighing roughly 5 $\mathrm{Kgs}$ and an additional payload of $2 \mathrm{kgs}$ that could be connected at the base of the UAV. The science payload would analyse both the Martian atmosphere and surface during flight.

The detailed flight plan is shown in the image.

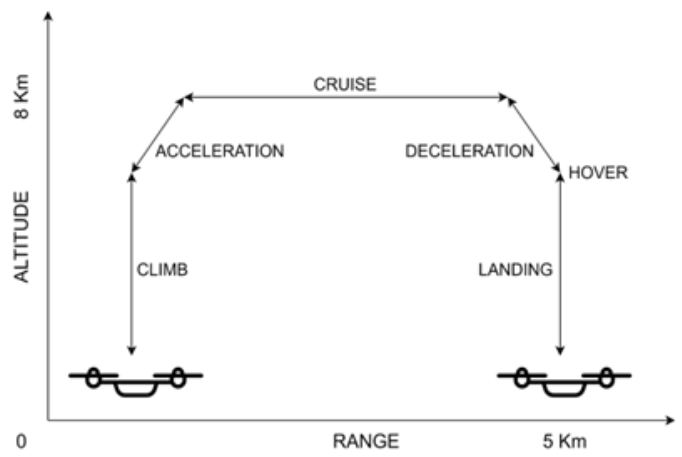

Fig. 1. Mission Architecture.

\section{MODES OF OPERATION}

The hybrid UAV consists of three modes of operation concerning flight stages. VTOL mode for vertical take-off and landing along with the inclusion of loitering as well at a specific altitude and location. The fixed-wing aircraft mode for normal flight operations (horizontal translation).

The transition and tuning mode for switching between VTOL and Fixed Wing mode.

\section{A. VTOL Mode}

This phase has been intended for vertical take-off, landing, and hovering operations. During this phase, the outboard wing panels spin generating sufficient thrust to lift the UAV. To save power consumption, the UAV platform automatically switches to aircraft mode after reaching the appropriate height.

\section{B. Transition Mode}

The thruster-mounted shaft of the UAV starts and provides a forward thrust. The two outboard wing panels are halted synchronously which causes a halt to the vertical motion of the UAV and the outboard panels now act as a fixed-wing. With an increase in horizontal speed, the fixed wings start to develop lift.

\section{Aircraft Mode}

During this phase, the UAV has reached a sufficient translational speed and is producing a sufficient lift force to offset its weight. Under these conditions, the platform performs like a typical airplane, with control surfaces such as ruddervators, ailerons, and flaps providing the necessary yaw, pitch, and roll motions. This phase is designed for energyefficient operation throughout a large chunk of the mission.

\section{DESIGN PROCESS}

A systematic design approach has been used to design our UAV. The constraints have been analysed first followed by the selection of aerofoils for both wing and tail. A preliminary design of the fuselage, wing, and tail has been developed based on calculations and analysis which has been refined to minimize weight and optimize storage. The final design has undergone several analyses to ensure whether or not the design is adept enough to fly in the challenging Martian atmosphere. Structural and Thermal analyses have been carried out to prevent any kind of mechanical failure during operations. As shown in Fig 2.

\section{UAV MECHANICS}

All of the materials used in the parts were chosen after careful consideration of different criteria such as weight, hardness, yield strength, tensile strength, and so on.

Owing to the property of gold to block radiation, the parts of the UAV have been gold plated to protect the UAV from extreme levels of UV Radiations.

After extensive investigation and testing of different alternatives, gear lubrication has been assured by a mixture of $50 \% 802 \mathrm{EP}$ grease and $50 \% 815 \mathrm{Z}$ oil to ensure efficiency even at extremely low temperatures since these lubricants do not dry up quickly. Penzane-3 Pb Np oil and $601 \mathrm{EF}$ have been produced from PFPE oil thickened with PTFE powder [2] for lubricating other components such as bearings, motors, and so on.

The aerodynamic and structural analysis of the UAV has been done considering several factors and the calculations have been made considering the weight of the UAV to be 10 Kgs on Mars.

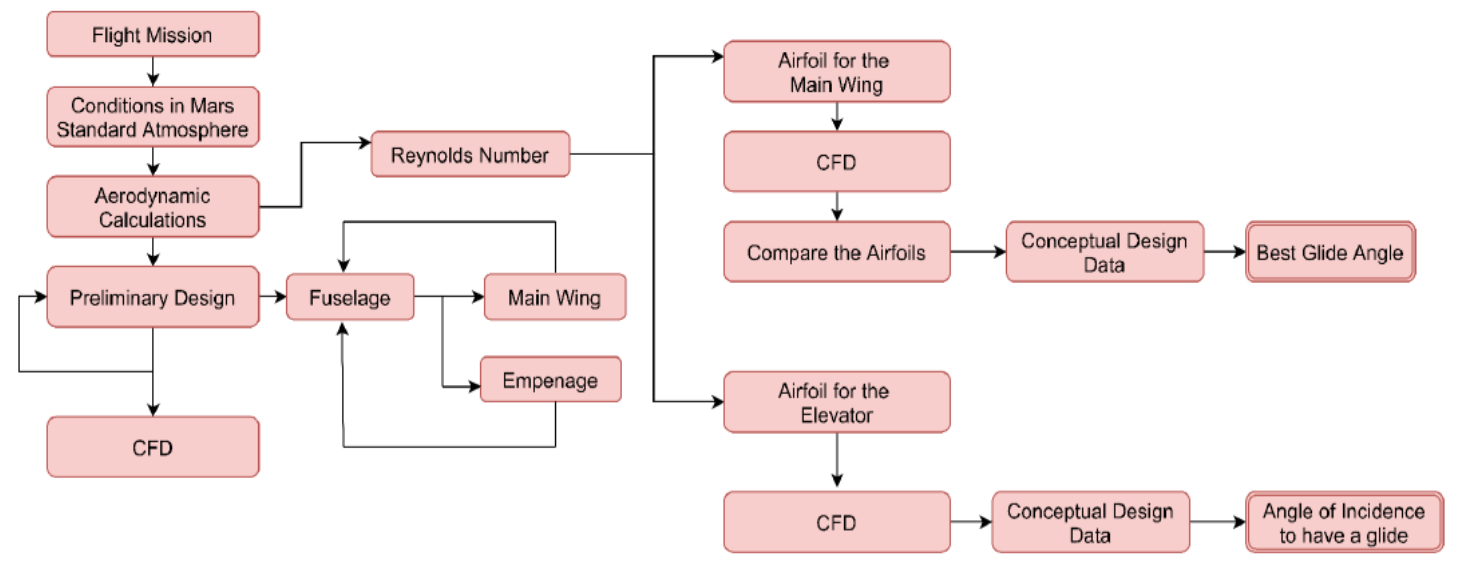

Fig. 2. Design Process. 


\section{GEOMETRY}

Our plan has mixed the drifting capacity of a helicopter and rapidly expanded the perseverance flying of a fixed-wing airplane. This has helped our UAV to gain the benefits of both into one.

We have used outboard wing panels that are $1 \mathrm{~m}$ long and have a root and a tip chord of $0.3 \mathrm{~m}$, that spin to generate thrust to lift the UAV in vertical flight. The wings rotate at 3000 RPM generating a maximum lift of $110 \mathrm{~N}$ making them capable of lifting the UAV from the ground. Both rotors rotate in opposite directions hence generating a contour torque preventing the UAV from spinning. During horizontal translation the outboard wing panels lock hence, acting as a fixed-wing. A pusher propeller is located aft of the UAV to provide thrust during the cruise. For deciding the wing geometry, possible planform shapes have been explored extensively, several trade studies between wing area and aspect ratio have been conducted, and the theoretical position of the wing aerodynamic centre has also been restricted to avoid excessive deviations from the needed aircraft centre of gravity. Based on the outcome of these studies, A baseline planform with a 17.4 aspect ratio and a wing size of $1.8 \mathrm{~m}^{2}$ has been chosen.

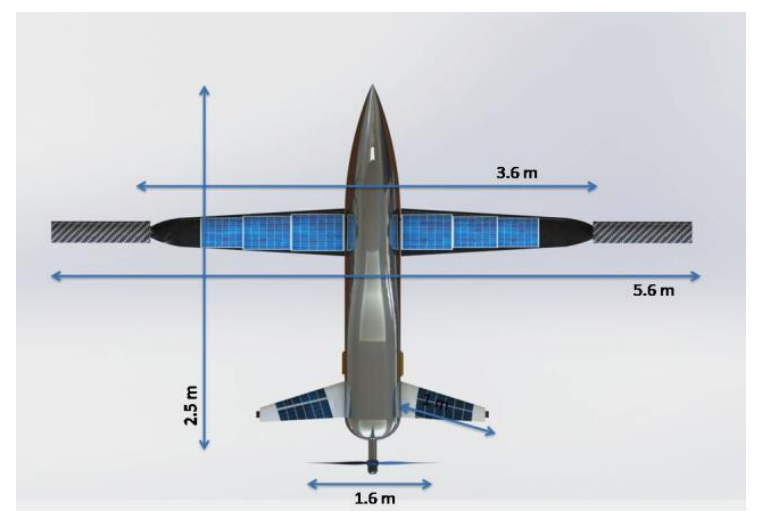

Fig. 3. Dimensions

On the ground, the plane is supported using a landing mechanism fabricated using Aluminium - 7079 at the front and on the Y-Tail at the aft position. The landing mechanism is connected to the plane using Heim joints providing flexibility to the structure making it capable of landing comfortably on uneven Martian terrain.

\section{FUSELAGE}

The Fuselage is an integral part of the UAV. It has been designed to maximize storage capacity while minimizing weight. The fuselage houses the Flight Electronics, Science Module, and everything that has to be incorporated in or on the UAV. The Fuselage is $1.5 \mathrm{~m}$ long and about $0.6 \mathrm{~m}$ wide making it adept to cache all the components appropriately, enabling a compact and efficient design. The Fuselage has been fabricated using Mylar and AS Graphite-Epoxy composite [3]. This material provides an excellent strengthto-weight ratio and could easily withstand both temperature extremes on Mars. Ease of Serviceability has been kept in mind while designing all the components making all subsystems accessible to the astronaut during maintenance. Openings have been provided both under the fuselage as well as on the rear end which makes it easier to access the electronics without dismantling the wings.

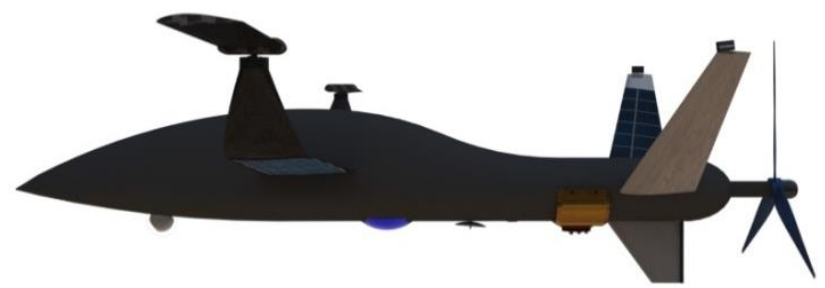

Fig. 4. Lateral View.

\section{AEROFOIL SELECTION}

Several Airfoils including Eppler 203, NACA 66(3)-418, RG-15[4], i.e., those capable of operating under Low Reynolds Number and High Mach Number (0.6-0.8) on the Martian atmosphere have been considered and after performing extensive analysis of those airfoils considering various key factors such as density, pressure, gravity, etc.

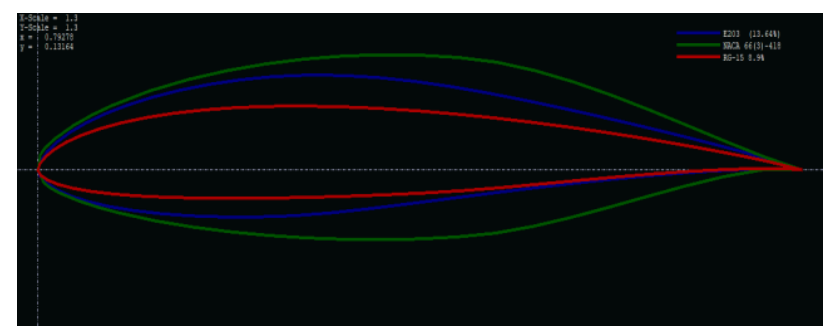

Fig. 5. Aerofoil Comparison.

RG-15 has been selected for our design. The aerofoil provides a minimum drag coefficient as well as a maximum lift coefficient, which means the highest lift-to-drag ratio; also, it provides a low pitching moment coefficient. These characteristics empower our UAV wing with noteworthy thrust and negligible drag, subsequently diminishing the power consumption and force utilization for an efficient flight. The aerofoil which has been selected for our UAV guarantees a positive Coefficient of Pressure enabling us to achieve a Lift Coefficient as high as possible. An angle of attack of $8 \mathrm{deg}$. has increased the aerodynamic force and has streamlined power, along with giving a positive moment about the Aerodynamic Centre.

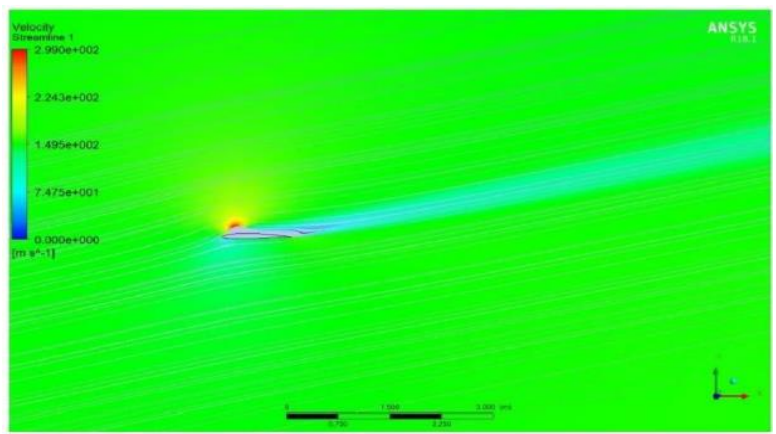

Fig. 6. Pressure and Velocity Graph of RG-15. 


\section{RIB DESIGN}

Morphed wing configuration has been for the UAVs fixed wings, which consists of three main components: aluminium profiles, low-temperature foam, and silicone elastomers.

\section{A. Low-Temperature Foam}

It has been used to fill the gap between the aluminum profiles. A protective layer of low-temperature silicone elastomers has been applied to aluminum profiles and foam [5].

\section{B. Skin Material}

Silica Elastomer has been used as the material for the skin hence making the morphed wing adaptable and flexible so that the material is handily twisted, while as yet having the solidarity to convey the streamlined loads of the UAV. Likewise, the material has been supplied with an abrasionresistant coating so that the material is not harmed when liable to evolving conditions. After the material has been converted to an alternate wing shape, the material recuperates its original size. The "skin" of the wing additionally upgrades the construction's exhibition. It has been produced using covering portions of adaptable material, layered to some degree like quills or fish scales, taking into account the pieces to get across one another as the wing flexes, while as yet giving a smooth external surface.

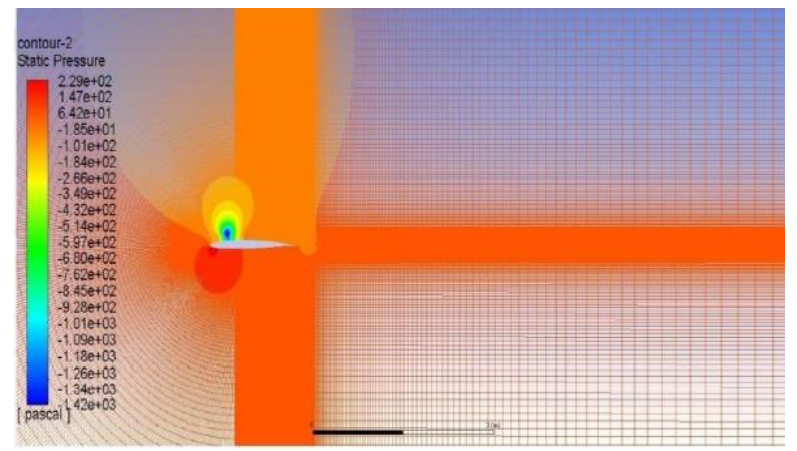

Fig. 7. Rib Structure.

\section{UAV Morphing Plan [6]}

The plan will affect the flying performance of our UAV by the dramatic change of wing shapes. Such plans take out the requirement for various, costly, mission-explicit aerofoils. Morphing aerofoils can change their shape for various flying performances. Such changes can be either incremental change to facilitate flight control. The key to the successful morphing of our wing was geometric scaling to match a particular performance requirement. The morphing would be carried out using 4 servo motors placed within the fixed-wing and connected to the spars. These ensure precise morphing to facilitate the required aerodynamic characteristics.

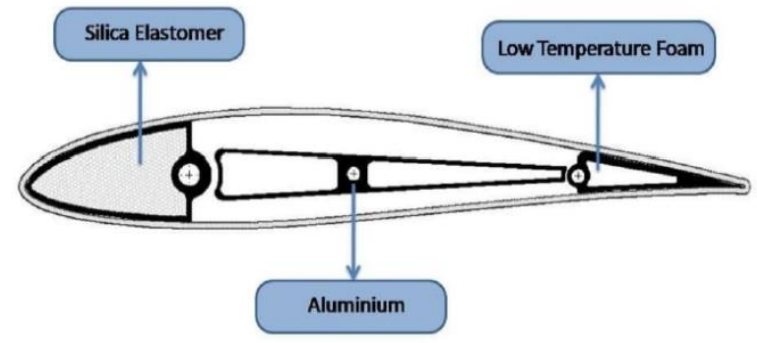

Fig. 8. Skin.

\section{EMPENNAGE DESIGN}

The Empennage provides for trim, stability, and control and is designed to operate normally at only a fraction of lift potential. Y- Tail configuration has been chosen for reducing drag interference, avoiding the complexities of ruddervators, and keeping the propeller off the ground when compared to conventional tail. The horizontal tail provides better stability and the vertical tail reduces aerodynamic sideslip and provides directional stability. NACA 0009, has been chosen for our UAVs tail which guarantees pitch control and longitudinal stability since it is an asymmetrical airfoil. This airfoil also provides a negative lift with an increased angle of attack, resulting in a negative moment, allowing the UAV to remain naturally stable.

TABLE I: EMPENNAGE DESIGN PARAMETERS

\begin{tabular}{ccc}
\hline Parameters & Horizontal Tail & Vertical Tail \\
\hline Root Chord & $0.4188 \mathrm{~m}$ & $0.25 \mathrm{~m}$ \\
Tip Chord & $0.1637 \mathrm{~m}$ & $0.15 \mathrm{~m}$ \\
Total Surface Area & $0.74 \mathrm{~m} 2$ & $0.344 \mathrm{~m} 2$ \\
Wing Span Length & $2.5408 \mathrm{~m}$ & $0.3 \mathrm{~m}$ \\
Wing Aspect Ratio & 8.71 & 1.60 \\
\hline
\end{tabular}

\section{PROPELLOR DESIGN}

The propeller blade has been designed for the lowest weight possible. Mylar, a light plastic material, has been chosen as the primary component of the skin structure. Because of its excellent strength-to-weight ratio, graphiteepoxy composite [7] was chosen as the material for the skinsupporting structure. The baseline design consists of a single hollow spar with rib elements to support the Mylar skin. To avoid aeroelastic instabilities, the blade's centre of gravity has been restricted to be coincident with the quarter chord axis position. NACA 16-012 aerofoil has been selected for our propeller. With a $70^{\circ}$ twist angle and desired minimum thickness of $12 \%$, our propeller ensures a high turning rate with a motor of 3000 RPM. The blade design is inherently smoother with reduced tip speed and minimal noise. This design marks constant thrust along the entire length of blades thereby maintaining the pitch to diameter ratio of $1: 1$ and making it more efficient.

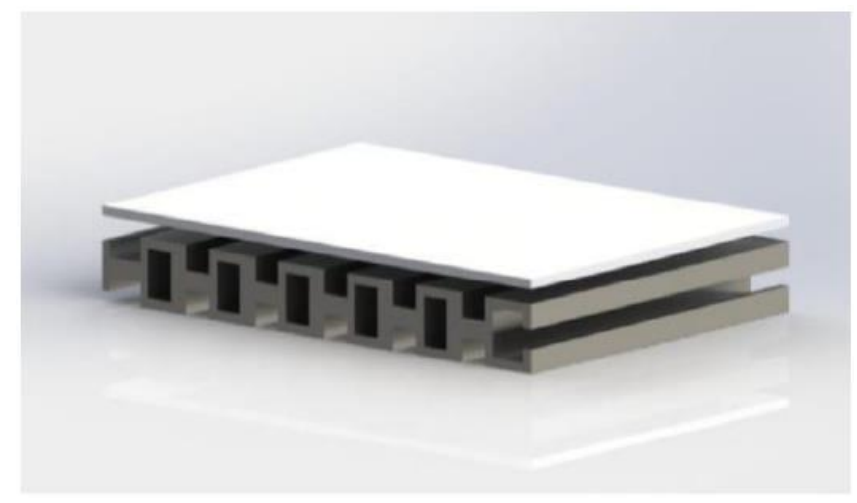

Fig. 9. Weight Distribution. 


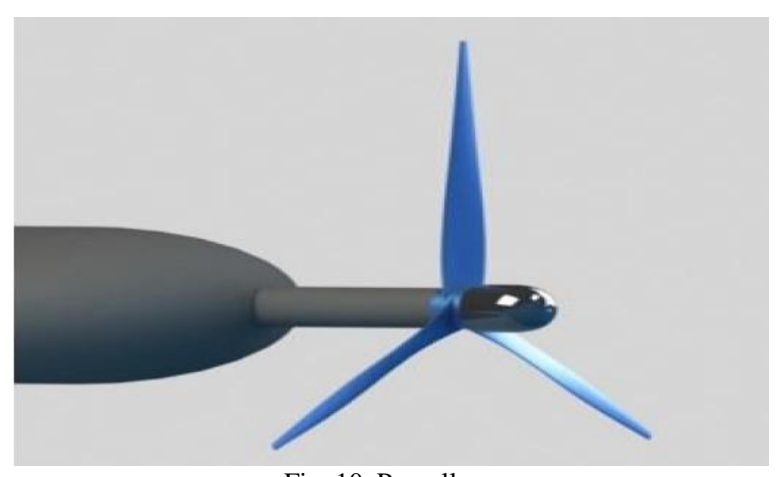

Fig. 10. Propellor.

\section{WEIGHT DISTRIBUTION AND BALANCING}

The well-known Boeing-Vertol equations were used in preliminary investigations to determine the weight breakdown. This algorithm takes size, gross weight, and other basic parameters and compares them to historical patterns. The output of these equations includes more detailed weight information on UAV subsystems.

\begin{tabular}{ccc}
\multicolumn{3}{c}{ TABLE II: WEIGHT DIVISION } \\
\hline S.no & Sub-System & Weight $(\mathrm{Kg})$ \\
\hline 1. & Structural & 4.0 \\
2. & Batteries and Propulsion & 1.2 \\
3. & Electrical & 0.3 \\
4. & Science Payload & 4.7 \\
& TOTAL & $\mathbf{1 0 . 2}$ \\
\hline
\end{tabular}

The design has been optimized using the data obtained to have a maximum scientific payload capacity without exceeding our weight. Our final weight estimates slightly deviated from the traditional Boeing-Vertol formulae due to the following reasons:

- Our fuselage is lighter than anticipated since our plane is unmanned. Composite materials have also been used throughout instead of traditional heavier metals.

- Our wings and blades have been made using composite materials hence are lighter than traditional designs. Hence, they were lighter than predicted.

\section{ENVIRONMENTAL CONTROLS}

\section{A. Temperature Management}

To make our UAV adaptable to the fluctuations in Martian temperatures from $20{ }^{\circ} \mathrm{C}$ to a freezing $-150^{\circ} \mathrm{C}$ we have introduced a wire meshing network in the main UAV body. The meshing is the primary heating system for the Electronics and Science subsystems. The network is connected to the centralized power system via a relay. The meshing is made of electrically insulating and thermally conductive diamond coating on copper coils [8] thus providing electrical safety to the components without compromising on thermal parameters of the devices used.

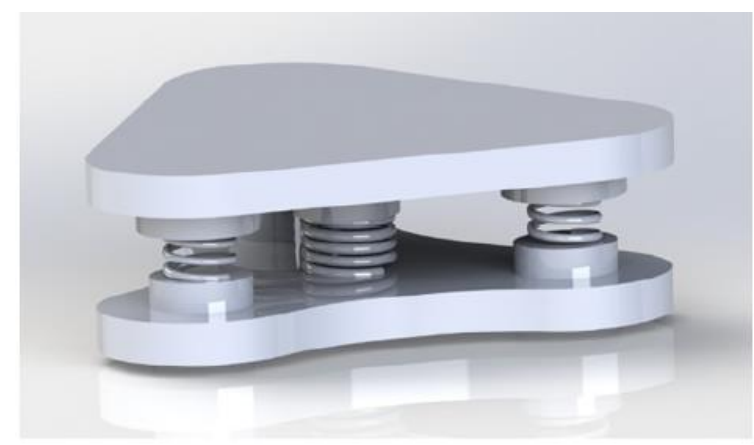

Fig. 11. Heat Switch.

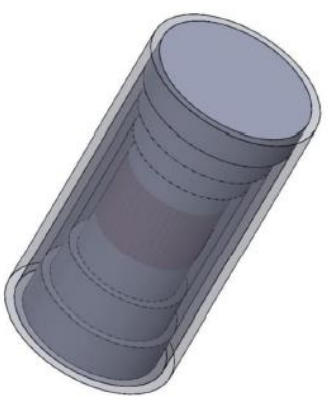

Fig. 12. RHU.

In addition to the meshing network, a robust Temperature Control System (TCS) has been incorporated. TCS is inclined towards sustainable temperature management without compromising any power consumption. It consists of 8 Radioisotope Heating Units (RHUs) [9] spread across the plane. RHUs have an excellent capability to produce heat and additionally provide the benefit of reduced electromagnetic interference to their electrical heating counterparts. It provides a constant supply of $1 \mathrm{~W}$ heat without adding on a burden on the power cache as they provide heat through radioactive decay. The placements of RHUs have been criticized due to the difference in the amount of heat required by individual components.

Adequate temperature range for each component is maintained using heat switches and insulation as discussed below:

Maximum heat insulation has been provided for the UAV to ensure it withstands cold Martian. All electronic components are placed within this insulated box known as Heating Module. It has been further thermally regulated using graphene tubing [10] perforated through the box which conducts heat through it to the base of the payload carrying mechanism. It prevents our UAV from overheating during operations on warm Martian days.

The radiators have been designed such that, in the worst scenario of temperature variation they maintain the optimal working temperature in the Heating Module. To prevent heat loss during the night, the conduction tubes would be connected to the radiator plates through Shape Memory Heat Switches [11]. These are metallic materials that can return to a previous shape or size when subjected to the right thermal conditions. These switches can operate within a temperature range of roughly $-200 \mathrm{C}$ to $+100{ }^{\circ} \mathrm{C}$, making them suitable for the Martian atmosphere. 


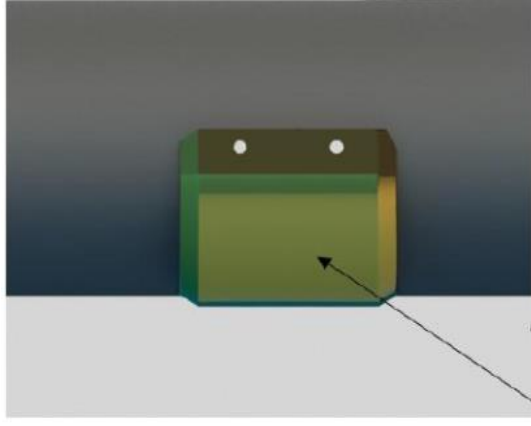

RADIATORS

Fig. 13. Radiators

To trap heat inside the UAV the inside of the UAV would be flushed with $\mathrm{CO} 2$ gas. Also, a layer of solid silica aerogel [12] would be placed around the electronic box and the motors. Both carbon dioxide and silica aerogel are excellent insulators of heat. These prevent any unwanted heat loss from the plane's surface. Graphene has been employed as the material for making the tubes as it has an excellent conduction and convection potential, hence making it an ideal material for making the tubes. To allow efficient heat transfer between radiators and the Martian atmosphere the radiators are made of copper which is an excellent conductor of heat.
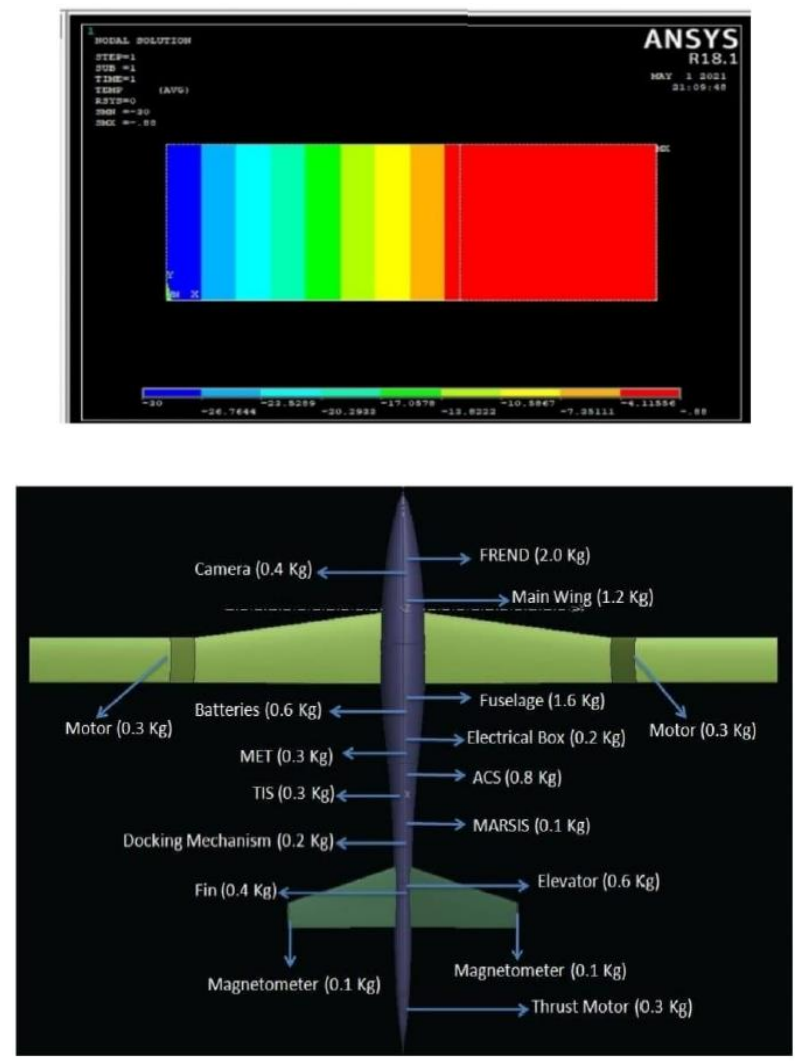

Fig. 14. Thermal Analysis of Fuselage.

\section{B. Dust Protection}

Mars is infamous for intense dust storms, which sometimes kick up enough dust to be seen by telescopes on Earth. Individual dust particles on Mars are very small and slightly electrostatic, so they stick to the surfaces they contact, and consequently, the possibility of dust settling on and in machinery is a challenge for the mission. To prevent this from happening the entire exposed surface of the UAV is covered with a dust repellent layer of CVD Nano-Coating ${ }^{[13]}$ that prevents the UAV surface from wear due to the bombardment of fast-moving dust particles. To protect the cameras and lenses from damage due to dust and wind, they are equipped with lens-protective covers.

\section{SCIENCE PAYLOAD}

The science payload for the UAV comprises the following components:

\section{A. Planetary Surface Analyzer (PSA)}

It is a CCD Camera and electronics kit with a dual optic path and a single focal plane scope. It would be used for providing regular summary and low-goal imaging of Mars to examine the climatic changes.

\section{B. Calorific Imaging Spectrometer (CIS)}

It is a plane reflection spectrometer that would be used for the analysis of the Martian atmosphere and to measure the surface composition of $\mathrm{CO}_{2}, \mathrm{~N}_{2}, \mathrm{Ar}, \mathrm{O}_{2}$, etc.

\section{Atmospheric Data System (ADS)}

It will provide a comprehensive measurement of the Martian near-surface atmosphere. Also, it would be used to measure the wind speed and direction.

\section{Infra-Red Spectral Chemistry Suite (ISCS)}

The primary goal of the ISCS instrument would be to detect minor atmospheric gases along with sensitive detection of minor gases and Isotopes to address global scientific issues related to Mars.

\section{E. High Precision Neutron Detector (HPND)}

It would help gather more information about the geology of Martian soil. It would also be used to create high-resolution maps of hydrogen-abundant regions on Mars.

\section{F. Subsurface Sounding Radar and Altimeter (SSRA)}

The primary goal of SSRA would be to map the distribution of water and ice in the upper layers of Martian Crust. It also acts as an altimeter.

\section{G. Magnetometer}

It will study Mars' magnetic and electric fields as well as its plasma environment.

\section{CONCLUSION}

We presented the conceptual aerodynamic design of a UAV in this article capable of performing a VTOL (Vertical Take-Off and Landing) in the atmosphere of Mars. The UAV is capable of traveling over a range of $10 \mathrm{~km}$ per flight at an altitude of $7-8 \mathrm{~km}$ above the surface. It is equipped with Cameras and Sensors that would be used to analyze the surface and atmosphere of Mars providing us with a better understanding of the Planet. It is capable of withstanding harsh Martian climate owing to its Temperature control and dust protection systems. Such UAVs will have a crucial role in future Mars missions as they provide us with a better overview of the Planet. 


\section{FUTURE SCOPE}

\section{A. Surface Mapping}

The UAV could be used for mapping the surface of Mars. The data so obtained could be used for planning further rover missions or to find a suitable site for humans to settle.

\section{B. Transportation}

The UAV could be used to transport essential items over a larger range faster.

\section{Exploration}

The UAV could be used to Explore hard-to-reach places and provide the required data for further analysis.

\section{Meteorology}

The UAV could be used to monitor regular changes in climatic conditions. Providing a better understanding of the Martian atmosphere.

\section{REFERENCES}

[1] William J. Fredericks, "Conceptual design of a VTOL Unmanned Aerial Vehicle with 24Hr Endurance".

[2] Robert L. Fusaro; "Lubrication of Space Systems".

[3] Wolf Elber; "Toughening of Graphite-Epoxy Composites by Interlaminar Perforated Mylar Films"; NASA March 1978.

[4] California State University Northridge; "Project Ares II: High-Altitude Battery Powered Aircraft"; Proceedings of the NASA/USRA Advanced Design Program 7th Summer Conference.

[5] Oliver Schorsch, Andreas Luhring, Christof Nagel et al., "Polymer Based Morphing Skin for Adaptive Wings".

[6] Hangil You, Seongik Kim, et al.; "New Concept for Aircraft Morphing Wing Skin: Design, Modeling, and Analysis". DOI: 10.2514/1.J058102

[7] Rudy Lukez; "The use of Graphite/Epoxy Composite Structures in Space Applications",

[8] ", Daichi Nakajimaa, Hiroki Kuwabara; "Diamond-like carbon coating for effective electrical insulation of $\mathrm{Cu}$ and $\mathrm{Al}$ wiresNASA Article on "Radioisotope Heater Unit".

[9] Yifeng $\mathrm{Fu}$; "Graphene related materials for thermal management" et al 2020 2D Mater. 7/01/2001.

[10] K. Lankford; "Heat Switches" Starsys Research Corporation, Boulder Colorado.

[11] J.E. Fesmire; "Aerogel Insulation Systems for Space Launch Applications."

[12] A. Delfini, A. Vricella, R. Bueno Morles." CVD nano-coating of carbon composite for Space materials." Procedia Structural Integrity Volume 3, 2017.

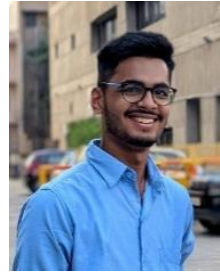

Ishan Mishra was born on 14th February 2001 in Dharan, Nepal. He is currently pursuing his B. Tech Degree in Mechanical Engineering from Delhi Technological University and is expected to complete his B. Tech in 2023.

He is currently working as the Head of Design in Team Inferno DTU which is a student-based technical team working on the design and fabrication of Mars Rover Prototypes. His areas of research include Robotics, Design of Unmanned Aerial Vehicles, Design of Mars Rovers, and Mars Explorations. He has presented a paper on "Smart Manufacturing: Opportunities and Challenges overcome by Industry 4.0" in the International Conference on Advanced Manufacturing and Materials Processing (CAMMP 2021) organized by MNIT Jaipur and a Journal paper on "Conceptual Design of a 7 DoF Robotic manipulator for Martian Applications" in IJISET Vol 8 Issue 6, 2021

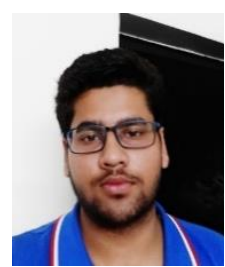

Aayush Kumar was born on $14^{\text {th }}$ August 2001 in Gurugram, Haryana. He is now studying his B.Tech Degree in Mechanical Engineering with minors in robotics and automation at Delhi Technological University, and he is anticipated to complete his B. Tech in 2023 .

He is presently the Mechanical Lead of Team Inferno DTU, a student-led technical team tasked with the design and fabrication of Mars Rover prototypes. His scientific interests include robotics, the design of the Mars Rover prototype, a 6 Degree of Freedom robotic arm manipulator, and his ongoing project include Conceptual design of fixed-wing UAVs unmanned aerial vehicles for both terrestrial and extra-terrestrial explorations his expertise lies in the field of fluid dynamics and finite element analysis.

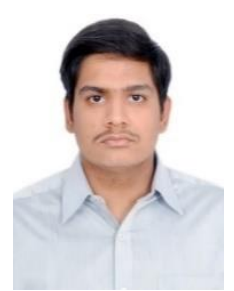

Vanshaj Malhotra was born on 24th May 2001, in Delhi India. He is currently pursuing his B.Tech Degree in Mechanical Engineering with Minors in Robotics and Automation from Delhi Technologica University and is expected to complete his B.Tech in 2023.

$\mathrm{He}$ is currently working as a Team Leader in Team Inferno DTU which is a student-based technical team working on the design and fabrication of Mars Rover Prototypes. His areas of research include Robotics, Design of Unmanned Aerial Vehicles, Design of Mars Rovers, and Mars Explorations. He has published a conference paper on "Role of Robotics in Mass Manufacturing and its Associated Advantages and Disadvantages" CAMMP 2021 organized by MNIT Jaipur and a Journal paper on "Conceptual Design of a 7 DoF Robotic manipulator for Martian Applications" in IJISET Vol 8 Issue 6, 2021. 